\title{
Optical third-harmonic generation in the fluorinated methanes and sulfur hexafluoride
}

\author{
J.F. Ward \\ The Harrison M. Randall Laboratory of Physics, University of Michigan, Ann Arbor, Michigan 48109 \\ D. S. Elliott
}

Joint Institute for Laboratory Astrophysics, University of Colorado and National Bureau of Standards, Boulder, Colorado 80309

(Received 23 May 1983; accepted 7 July 1983)

Optical third-harmonic generation experiments are used to determine nonlinear polarizabilities for the fluorinated methanes and sulfur hexafluoride. An ambiguity present in previous measurements of thirdharmonic generation in gases is resolved.

We wish to report measurements of optical third-harmonic coefficients for the fluorinated methanes and sulfur hexafluoride.

The nonlinear optical properties of essentially noninteracting molecules in gases were first measured using optical third-harmonic generation ${ }^{1}$ and the Kerr effect. ${ }^{2,3}$ Subsequently dc electric-field induced second-harmonic generation (dcSHG) $)^{4-7}$ became the preferred technique. These data have stimulated theoretical evaluations of the coefficients, involving techniques at the forefront of molecular calculations. Nonlinear coefficients are often calculated in the static limit and compared with experimental coefficients measured in any of the above mentioned processes by estimating corrections for electronic dispersion. ${ }^{8}$ However, interesting differences between nonlinear polarizabilities for different processes have been noted, differences which are not subject to explanation by electronic dispersion alone. ${ }^{9}$ The role of vibrational contributions to second-order nonlinear polarizabilities has been discussed in this connection. ${ }^{10-12}$

The present measurement of third-harmonic polarizabilities for $\mathrm{CH}_{4}, \mathrm{CH}_{3} \mathrm{~F}, \mathrm{CH}_{2} \mathrm{~F}_{2}, \mathrm{CHF}_{3}, \mathrm{CF}_{4}$, and $\mathrm{SF}_{6}$ was stimulated by the need to use these data together with existing deSHG ${ }^{4-6}$ and $\mathrm{Kerr}^{2,3}$ data to investigate vibrational contributions to third-order polarizabilities. Progress in experimental techniques since Ref. 1 also adds interest to this measurement.

In this paper, we briefly review the experimental procedure for measuring the third-harmonic polarizability and present our results. These data are compared with third-order dcSHG and Kerr polarizabilities. A detailed analysis of dispersion effects, including evidence for contributions from the molecular vibrational modes, is described elsewhere. ${ }^{13}$

The optical arrangement used here for the measurement of third-harmonic coefficients is shown in Fig. 1 and is similar to that used in Ref. 1. Improved data acquisition ${ }^{4}$ has greatly increased the amount of data collected. In addition to reducing uncertainties, this allows ambiguities present in the results of Ref. 1 to be resolved.

The ruby laser beam (frequency $\omega=2 \pi c / 694 \mathrm{~nm}$, power
$P^{\omega}=1 \mathrm{MW}$, pulse length $40 \mathrm{~ns}$ ) is focused with confocal parameter $b=6.1 \mathrm{~mm}$ close to a glass/gas interfacesee Fig. 1. The glass is selected to be absorbing to third-harmonic radiation. The magnitude and phase of the harmonic radiation generated in the gas is density dependent, and interferes with the harmonic radiation generated in the last absorption length of the glass. The resultant harmonic intensity is detected upon exiting the cell. Since the cell is about 11 confocal parameters in length the fundamental beam is large enough at the quartz window so that harmonic generation there and subsequently is insignificant. A third-harmonic signal $s$ may be defined as the ratio of harmonic power to the cube of the fundamental power:

$$
S(\Omega)=P^{3 \omega}(\Omega) /\left[P^{\omega}\right]^{3} .
$$

The experiment consists of measuring $S$ as a function of $\Omega$, a normalized gas density defined by

$$
\Omega=\rho \Delta k_{0} b / 2,
$$

where $\rho$ is the gas density in amagats and $\Delta k_{0}$ is the wave number mismatch of the gas at a density of 1 amagat and is related to the refractive index differences $n^{\omega}-n^{3 \omega}$ :

$$
\Delta k_{0}=3 \omega\left[n^{\omega}(\rho)-n^{3 \omega}(\rho)\right] / c \rho .
$$

Typical data for $S(\Omega)$ is shown in Fig. 2. In order to calibrate the third-harmonic coefficient of each molecule against that of helium, alternate runs were carried out for helium and the gas under observation. Each run consisted of measurements of $S(\Omega)$ at ten values of $\Omega$ be tween 0 and 1.5 with 20-40 laser hosts at each value. Error flags in Fig. 2 indicate the standard deviation of

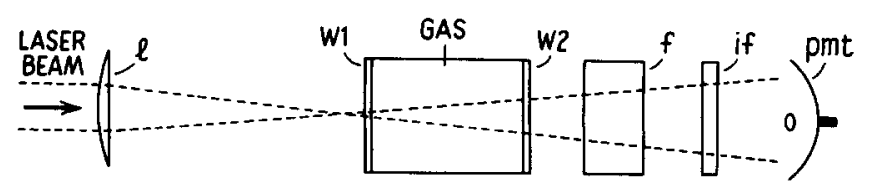

FIG. 1. Schematic diagram of the optical apparatus. The elements are labeled as follows: $l: 16.5 \mathrm{~cm}$ focal length lens, $\mathrm{w}_{1}$ : borosilicate glass window, $\mathrm{w}_{2}$ : quartz window, f: aqueous nickel sulfate filter, if: interference filter (peak transmission at $230 \mathrm{~nm}$, pmt: 1 P28 photomultiplier. 

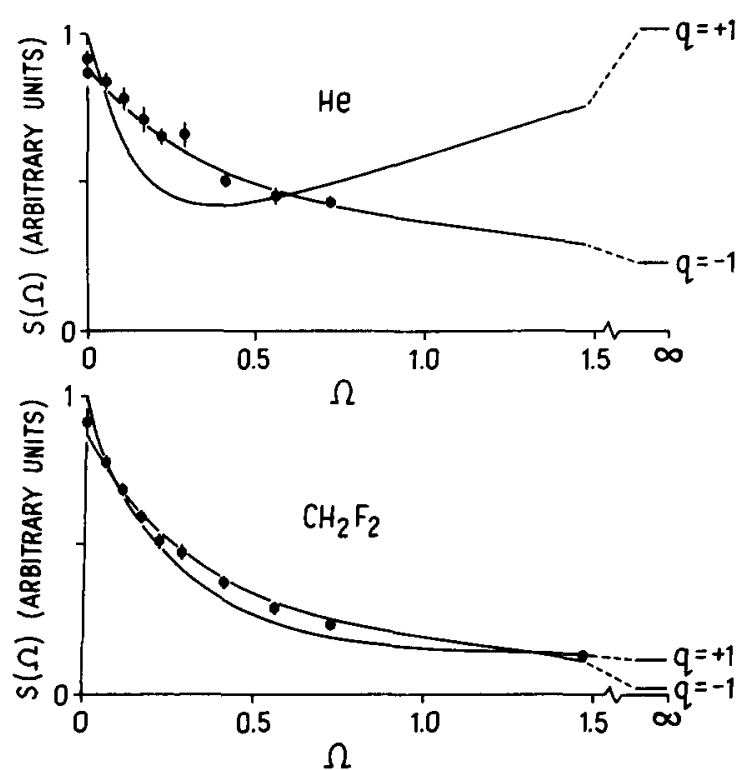

FIG. 2. Third-harmonic signal $S$ as a function of normalized gas density $\Omega$ for He (top) and $\mathrm{CH}_{2} \mathrm{~F}_{2}$ (bottom). The point at $\Omega=1.47$ for He is omitted since the pressure (about $200 \mathrm{psi}$ ) exceeds the capability of the gas cell. The two solid curves and extrapolations to infinite density for each gas are fits to Eq. (7) for each of the assignments: $c \cdot \chi /\left(-\Delta k_{0}\right)$ greater than unity $(q=+1)$ and $C \cdot \chi /\left(-\Delta k_{0}\right)$ less than unity $(q=-1)$.

the mean of $S(\Omega)$. These uncertainties arise largely from photon statistics [S(0) was typically 200 photoelectrons/ shot] and from fluctuations due to residual self-focusing in the glass window.

$P^{3 \omega}$ can be increased by bringing the fundamental beam focus closer to the glass/gas interface, but this also increases the risk of damage at the glass surface. Self focusing in the glass evidenced by a stronger than cubic dependence of $P^{3 \omega}$ on $P^{\omega}$, is also seen if the focus is too close to the glass window. A compromise position is chosen.

Analysis ${ }^{1}$ based on treatment of the fundamental beam as a lowest order Guassian mode, establishes a relation between $S(\Omega)$ and $\chi$, the third-harmonic coefficient ${ }^{1}$ of the normally dispersive gas:

$$
S(\Omega)=S(0)\left|1-C \cdot \chi /\left(-\Delta k_{0}\right) \cdot Q\left(\Omega, z_{I}\right)\right|^{2},
$$

where $C$ includes parameters relating to the glass, and can be shown to be real ${ }^{1}$ and $Q\left(\Omega, z_{1}\right)$ is a complex integral, depending on beam geometry and gas density given by

$Q\left(\Omega, z_{I}\right)=-i \Omega\left(1+i 2 z_{I} / b\right) \int_{2 \alpha_{I} / b}^{\infty} \frac{d \xi \exp \left[i \Omega\left(\xi-2 z_{I} / b\right)\right]}{(1+i \xi)^{2}}$,

$Q\left(\Omega, z_{l}\right)$ for general $\Omega$ must be evaluated numerically and its limiting values are

$$
Q\left(0, z_{I}\right)=0 ; \quad Q\left(\infty, z_{I}\right)=1,
$$

where $z_{I}$ is the equivalent free-space focus/interface distance. Thus, Eq. (4) can be rewritten:

$$
S(\Omega)=S(0)\left|1-\{1+q \sqrt{S(\infty) / S(0)}\} Q\left(\Omega, z_{l}\right)\right|^{2},
$$

where

$$
q= \pm 1
$$

is introduced to represent the sign ambiguity which corresponds in Eq. (4) to $C \cdot \chi /\left(-\Delta k_{0}\right)$ less or greater than unity. The solid lines in Fig. 2 are fits to the data for each of the two values of $q$. The fitting parameters are $S(0)$ and $S(\infty)$. In some cases-specifically He (see Fig. 2) and $\mathrm{SF}_{6}$ - the relative quality of fit for the two values of $q$ leads to a definite choice of $q$. In other cases such as $\mathrm{CH}_{2} \mathrm{~F}_{2}$ (see Fig. 2), the difference in quality of fit is less clear. In all cases where a choice based on it is possible, the result is $q=-1$, i. e., $C \cdot \chi /$ $\left(-\Delta k_{0}\right)<1$. Additional evidence concerning the choice of $q$ is obtained here as in Ref. 1 by considering binary mixtures of gases.

The harmonic signal generated by a gas mixture can be predicted from $S(0), S(\infty)$, and $q$ for each gas together with $Q\left(\Omega, z_{1}\right)$. One signal value is predicted for equal $q$ 's and a different value of unequal $q$ 's. A series of mixture experiments was performed, including all gases of interest here at least once. Comparing experimental signals with predictions showed clearly that, in every case, the $q$ 's for the two gases were equal. Together with the quality of fit results [that $q(\mathrm{He})=q\left(\mathrm{SF}_{6}\right)=-1$ ], this establishes that $q=-1$ for all gases studied here. Similar mixture experiments in Ref. 1 determined that $q$ was the same for all gases studied there, and indirect arguments were used to indicate that all were probably $q=-1$. Since He was one of those gases, our present results determine rigorously that $q=-1$ for all gases studied in Ref. 1.

The third-harmonic coefficient for each gas $\chi_{x}$ is measured relative to helium for which a reliable theoretical value is available ${ }^{14}$

$$
\chi_{\mathrm{He}}=4.00 \times 10^{-39} \mathrm{esu} / \mathrm{atom} \text {. }
$$

Equation (4) with sign ambiguities resolved, yields:

$\chi_{\mathrm{X}}=\chi_{\mathrm{He}} \cdot \frac{\left(\Delta k_{0}\right)_{X}}{\left(\Delta k_{0}\right)_{\mathrm{He}}} \cdot \frac{1-\left[S_{X}(\infty) / S_{X}(0)\right]^{1 / 2}}{1-\left[S_{\mathrm{He}}(\infty) / S_{\mathrm{He}}(0)\right]^{1 / 2}}$.

Values for $\Delta k_{0}$ are known ${ }^{15}$ so that the fitted parameters $S(0)$ and $S(\infty)$ inserted in Eq. (10) yield the third-har monic polarizabilities shown in Table I.

\begin{tabular}{|c|c|c|c|c|}
\hline & \multirow[b]{2}{*}{$\chi^{2}$} & \multicolumn{2}{|c|}{$x(\mathrm{dcSHG})$} & \multirow[b]{2}{*}{$x($ Kerr) } \\
\hline & & & $b$ & \\
\hline$\overline{\mathrm{CH}_{4}}$ & $330(10)$ & $263(3)^{c}$ & $333(1)$ & $242(12)^{d}$ \\
\hline $\mathrm{CH}_{3} \mathrm{~F}$ & $258(10)$ & $239(30)^{\bullet}$ & $216(22)$ & \\
\hline $\mathrm{CH}_{2} \mathrm{~F}_{2}$ & $210(7)$ & $154(11)^{\bullet}$ & & \\
\hline $\mathrm{CHF}_{3}$ & $158(6)$ & $136(6)^{\circ}$ & $146(7)$ & \\
\hline $\mathrm{CF}_{4}$ & $99(5)$ & $91(3)^{\circ}$ & $103(1)$ & $125(7)^{d}$ \\
\hline $\mathrm{SF}_{6}$ & $148(9)$ & $130(2)^{t}$ & $144(1)$ & $200(16)^{2}$ \\
\hline
\end{tabular}

TABLE I. Third-harmonic coefficients $\chi \times 10^{39}$ (esu/molecule) compared with coefficients for dc electric-field induced secondharmonic generation, $\chi(\mathrm{dcSHG})$, and the Kerr effect $\chi(\mathrm{Kerr})$. Fundamental wavelengths used are indicated in the footnotes. 
Extraction of $S(0)$ and $S(\infty)$ by fitting Eq. (7) to the experimental data requires that $Q\left(\Omega, z_{I}\right)$ be evaluated numerically. $Q\left(\Omega, z_{I}\right)$ depends on the value assigned to $z_{I}$ (the effective free-space focus/interface distance) which is experimentally determined to be $(10 \pm 2) \mathrm{mm}$. Fitted curves in Fig. 2 correspond to $z_{I}=10 \mathrm{~mm}$. The fitting procedure was repeated for $z_{I}=12$ and $8 \mathrm{~mm}$ and while the effect on $S(0)$ and $S(\infty)$ for a single gas is significant, this is compensated by the corresponding changes in the helium fit and the resultant uncertainty in $\chi$ is never more than $5 \%$.

The values of $\chi$ may be compared in Table $\mathrm{I}$, with results for the same molecules as determined in dc electric-field induced second-harmonic generation $\chi$ (dcSHG), and the Kerr effect $\chi($ Kerr $)$. The Kerr effect third-order polarizabilities have not been determined for the nontetrahedral fluoromethanes since this term contributes comparatively little in the presence of orientational effects for these molecules.

In closing, we note that for similar frequencies the relation $\chi>\chi(\mathrm{dcSHG})>\chi(\operatorname{Ker} r)$, predicted on the basis of electronic dispersion ${ }^{8}$ is verified for $\mathrm{CH}_{4}$, but this relation does not hold for the molecules $\mathrm{CF}_{4}$ and $\mathrm{SF}_{6}$. It is argued in Ref. 13 that this indicates the existence of significant vibrational contributions.

${ }^{1}$ J. F. Ward and G. H. C. New, Phys. Rev, 185, 57 (1969).

${ }^{2}$ A. D. Buckingham and D. A. Dunmur, Trans. Faraday Soc. 64, 1776 (1968).

${ }^{3}$ A. D. Buckingham and B. J. Orr, Trans, Faraday Soc. 65, 673 (1969).

4J. F. Ward and I. J. Bigio, Phys. Rev. A 11, 60 (1975).

${ }^{5}$ C. K. Miller and J. F. Ward, Phys, Rev. A 16, 1179 (1977).

${ }^{6}$ J. F. Ward and C. K. Miller, Phys. Rev. A 19, 826 (1979).

${ }^{7}$ D. P. Shelton and A. D. Buckingham, Phys. Rev. A 26, 2787 (1982).

${ }^{8}$ E. L. Dawes, Phys. Rev. 169, 47 (1968); R. S. Finn, Ph. D. thesis, University of Michigan, Ann Arbor, 1971.

${ }^{9} \mathrm{G}$. Hauchecorne, F. Kerhervé, and G. Mayer, J. Phys. (Paris) 32, 47 (1971); R. S. Finn and J. F. Ward, J. Chem. Phys. 60, 454 (1974).

${ }^{10}$ B. J. Orr, Non-Linear Behavior of Molecules, Atoms and Ions in Electric, Magnetic or Electromagnetic Fields, edited by L. Neel (Elsevier, Netherlands, 1979), pp. 227-236.

${ }^{11} \mathrm{~B}$. J. Orr (private communication).

${ }^{13}$ D. M. Bishop, Mol. Phys. 42, 1219 (1981).

${ }^{13}$ D. S. Elliott and J. F. Ward, Mol. Phys. (to be published).

${ }^{14}$ P. Sitz and R. Yaris, J. Chem. Phys. 49, 3546 (1968).

${ }^{15}$ D. S. Elliott and J. F. Ward, JOSA Lett. (to be published). 